IRRITABLE BOWEL SYNDROME

\title{
Alteration of the spinal modulation of nociceptive processing in patients with irritable bowel syndrome
}

\author{
B Coffin, D Bouhassira, J-M Sabaté, L Barbe, R Jian
}

Gut 2004;53:1465-1470. doi: 10.1136/gut.2003.031310

See end of article for authors' affiliations

......................

Correspondence to:

Dr B Coffin, Service

d'Hépato-Gastroentérol ogie, Hôpital Lovis

Mourier, 178 rue des

Renovillers, 92701

Colombes Cedex, France;

benoit.coffin@

Imr.ap-hop-paris.fr

Revised version received 10 February 2004

Accepted for publication

3 March 2004

\begin{abstract}
Background: Visceral hypersensitivity has been evidenced in patients with irritable bowel syndrome (IBS) but its mechanisms remain poorly elucidated. We investigated the spinal transmission of nociceptive signals in IBS patients by analysing the effects of rectal distensions on electromyographic recordings of the somatic nociceptive flexion (RIII) reflex, an objective index of spinal nociceptive processes.

Methods: Fourteen IBS and 10 healthy volunteers were included in the study. Slow ramp $(40 \mathrm{ml} / \mathrm{min})$ and rapid phasic $(900 \mathrm{ml} / \mathrm{min}, 10,20,30$, and $40 \mathrm{~mm} \mathrm{Hg}$ ) rectal distensions were randomly performed while the RIII reflex evoked by electrical stimulation of the sural nerve at the ankle was continuously recorded from the ipsilateral biceps femoris.

Results: In healthy volunteers, significant progressive inhibition of the RIll reflex was observed during slow ramp distension (61 (13)\% of control values) while biphasic effects (facilitation and inhibition) were observed during rapid distensions. In contrast, in IBS patients, the RIII reflex was significantly facilitated during slow ramp distension (139 (15)\% of control values) and inhibitions induced by rapid distensions were significantly reduced. Volumes of distension and rectal compliance were similar in both groups.

Conclusions: Our results provide direct evidence that a hyperexcitability of spinal nociceptive processes is present in a large subgroup of IBS patients.
\end{abstract}

A bdominal pain is a major symptom of both functional and organic gastroenterological disorders. Irritable bowel syndrome (IBS), the most common gut functional syndrome, is characterised by abdominal pain or discomfort in association with bloating and/or defecation disorders and/or altered bowel habits. ${ }^{1}$ Hypersensitivity to physiological or experimental visceral stimuli (that is, reduction of pain or discomfort thresholds) has been repetitively demonstrated in these patients and is considered a major clinical feature of IBS. ${ }^{2}$ The pathophysiology of such a visceral hypersensitivity is probably multifactorial, involving both peripheral and central neural mechanisms, but still remains poorly understood. This concept has been challenged as it is mainly based on indirect evidence provided by measurements of subjective pain thresholds evoked by experimental visceral stimuli. ${ }^{3}$ There are still few objective data demonstrating hyperexcitability of pain systems in IBS patients although alteration of the processing of visceral sensory information has been suggested in electrophysiological $^{4-6}$ and functional neuroimaging studies. ${ }^{7}$

The aim of the present study was to investigate modulation of spinal transmission of nociceptive signals in IBS patients. We used an electrophysiological approach based on recordings of the somatic nociceptive cutaneomuscular flexion (RIII) reflex. The threshold and amplitude of this polysynaptic spinal reflex, elicited by electrical stimulation of a cutaneous sensory nerve and recorded from a flexor muscle on the ipsilateral limb, are closely related to those of the concomitant painful sensation evoked by electrical stimuli. ${ }^{8}$ Thus this reflex response is considered a reliable psychophysiological index of the spinal nociceptive processes in humans and has been used in numerous studies on the pharmacology and modulation of transmission of nociceptive signals. ${ }^{9}$ This methodology has been used notably to investigate alterations in pain modulatory systems in patients with fibromyalgia, chronic headache, and neuropathic pains. ${ }^{910}$ In a series of previous studies in healthy volunteers, we showed that the RIII reflex is strongly modulated by visceral stimuli (that is, gastric or rectal distensions) and that such an approach might be useful to investigate objectively and quantitatively processing of visceral sensory information in humans. ${ }^{11-15}$ In particular, we evidenced differential effects of rapid and slow ramp rectal distensions on the RIII reflex recorded from the lower limb, suggesting activation of two functionally distinct populations of mechanoreceptors by these two modes of distension..$^{12} 1415$

In the present study, we used a similar approach to compare the effects of rectal distensions on electrophysiological recordings of the RIII reflex in IBS patients and in healthy volunteers.

\section{METHODS}

\section{Participants}

Seventeen IBS patients (14 women; age range 31-76 years; mean 51 (13) years) recruited in a tertiary referral centre and 10 healthy volunteers (seven women; age range 30-46 years; mean 37 (6) years) participated in the study.

IBS patients were eligible if they met the Rome I criteria for IBS, including recurrent abdominal pain and bowel dysfunction for at least three months during the previous year. ${ }^{16}$ Each patient underwent a complete evaluation to exclude organic disease, including a detailed medical history and physical examination and a normal total colonoscopy within the five years preceding inclusion. None of these patients had past abdominal surgery, except for appendectomy. Antispasmodics, laxatives, and/or antidiarrhoeal agents were stopped at least 15 days before the experimental protocol. Seven patients were classified as constipation predominant IBS, five as diarrhoea predominant IBS, two had alternating bowel disorders while three had normal bowel movements. During the week before the experimental study, IBS patients were asked to report prospectively the mean daily intensity of abdominal pain and bloating on visual analogue scales

Abbreviations: IBS, irritable bowel syndrome; RIII reflex, somatic nociceptive cutaneomuscular flexion reflex 
(ranging from 0 (no pain) to 100 (intense pain)) and the number of bowel movements; on the experimental day, intensity of abdominal pain and bloating was also noted on a visual analogue scale.

Healthy volunteers had a normal physical examination, and no history of gastrointestinal symptoms or abdominal surgery, except for appendectomy. They were not allowed to take any medication during the two weeks preceding the experimental day, except for oral contraception.

Each participant gave written informed consent to the protocol that was approved by the ethics committee of SaintLouis Hospital (Paris, France).

\section{Nociceptive flexion reflex (RIII reflex)}

The RIII reflex was elicited and recorded from the lower limb according to a previously described and validated technique. ${ }^{11-15}$ Briefly, subjects were placed in the lateral decubitus position, and the RIII reflex was elicited and recorded by an entirely computerised system (Physio Labo System, Notocord, Croissy, France). The sural nerve was electrically stimulated at a frequency of $0.17 \mathrm{~Hz}$ (10 stimulations/minute) via a pair of surface electrodes placed $2 \mathrm{~cm}$ apart on the degreased skin overlying the nerve within its retromalleolar path. Each electrical stimulation consisted of a train of five constant current pulses of $1 \mathrm{~ms}$ duration. Electromyographic responses were recorded from the ipsilateral biceps femoris via a pair of surface electrodes placed $2 \mathrm{~cm}$ apart on the degreased skin over the muscle. The RIII response was identified as a multiphasic signal appearing between 90 and $180 \mathrm{~ms}$ after each stimulation. After amplification, each reflex response was digitised, full wave rectified, and integrated. This integrated surface was used to quantify the RIII response. The RIII reflex threshold was defined as the average minimal current that elicited the reflex response. Before each experiment, this threshold was determined by four successive sequences of increasing and decreasing electrical stimuli. The intensity of electrical stimulation of the sural nerve was then adjusted to $20 \%$ above the threshold and kept constant during the control, distension, and postdistension periods of each experimental sequence.

\section{Rectal distension}

An oversized spherical polyvinyl bag $(10 \mathrm{~cm}$ diameter, infinite compliance until maximal volume of $600 \mathrm{ml}$ ) was mounted on the tip of a double lumen polyvinyl tube (12 Fr) and inserted into the rectum. The proximal opening of the tube was linked to an electronic barostat (INRA, Toulouse, France) that allowed controlled inflation and deflation of the balloon with air and continuous monitoring and recording of the volume and pressure inside the balloon. When in place, the balloon was unfolded by slowly injecting air under controlled pressure $(<20 \mathrm{~mm} \mathrm{Hg})$ and then completely deflated. After a 20 minute period of rest, the barostat was used to inflate the balloon either rapidly $(900 \mathrm{ml} / \mathrm{min})$ to a constant pressure plateau (rapid phasic distension) or continuously at a constant volume rate of $40 \mathrm{ml} / \mathrm{min}$ (slow ramp distension). ${ }^{12}$ At the end of each phasic distension, the deflation rate of the balloon until $0 \mathrm{ml}$ was similar to the inflation rate while at the end of slow ramp distension the balloon was rapidly deflated $(900 \mathrm{ml} / \mathrm{min}$ ) until $0 \mathrm{ml}$.

\section{Perception of rectal distensions}

Before the experiments, participants were informed of the visceral sensation they might experience. The sensation elicited by rectal distension was graded from 0 to 6 using a verbal questionnaire previously validated ${ }^{12}{ }^{14}{ }^{15}: 0$, no perception; 1 , initial perception; 2 , sensation of gas; 3 , sensation of stool; 4 urge to defecate or onset of discomfort; 5 , moderate pain; and 6 , intense or unbearable pain. In the case of rapid distension, participants reported their sensations at the end of each distension period, and during slow ramp distension they reported their sensations at fixed intervals (every $50 \mathrm{ml}$ of distension). Distensions were performed until the threshold of moderate pain (score 5). Whenever intense or unbearable painful sensations (score 6) were experienced during any level of distension, the experiment was immediately suspended.

\section{Experimental design}

Experiments were performed after a 12 hour fast in a quiet room where patients were relaxed. Experimental sessions began with determination of the RIII reflex response; thereafter slow ramp and rapid phasic distensions were performed in a randomised manner.

Rapid distensions were performed at four levels $(10,20,30$, and $40 \mathrm{~mm} \mathrm{Hg}$ ). Each level was applied once and the order of application was randomised. Each distension was maintained for three minutes, and 10 minutes elapsed between application of each distension to avoid sensitisation phenomena. The RIII reflex responses were measured during the three minutes before distension (control period), during the three min distension period, and during the three minutes following distension (post-distension period). For each level of distension, the RIII responses were averaged at one minute intervals and expressed as a percentage of the mean control value. Mean volume was calculated for each distension step.

Slow ramp distensions were performed up to the pain threshold (score 5) or the maximal volume of the balloon
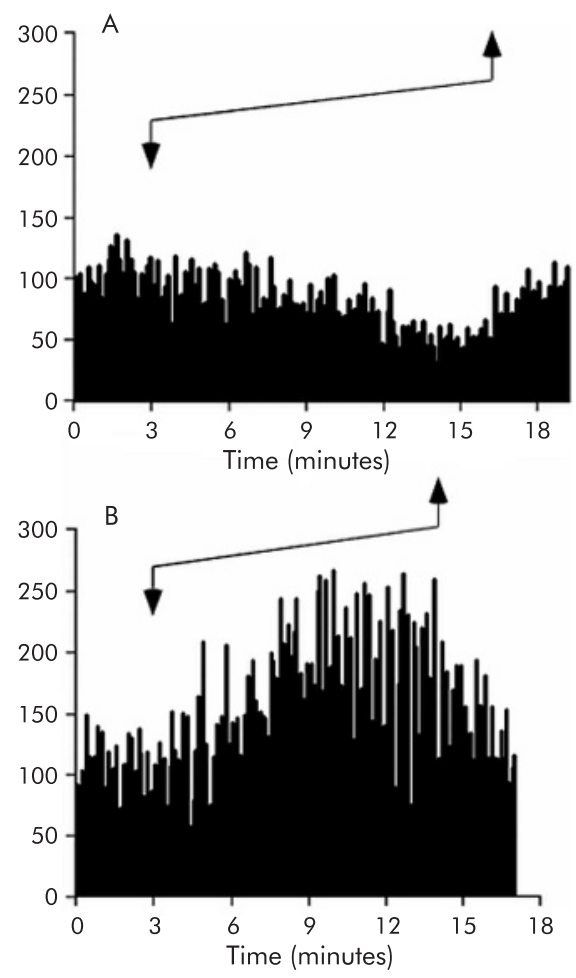

Figure 1 Individual examples showing the differential effects of slow ramp distension on the somatic nociceptive cutaneomuscular flexion (RIII) reflex recorded from the lower limb in healthy volunteers (A) and patients with irritable bowel syndrome (IBS) (B). Each bar represents a single reflex response expressed as a percentage of the mean value for the three minute pre-distension period. The period of increasing volume is indicated by arrows. (A) In healthy volunteers the reflex responses were progressively inhibited during distension, with a progressive return to baseline in the post-distension period. (B) In contrast with volunteers, in IBS patients the reflex was strongly facilitated during distension with a progressive return to baseline during the post-distension period. 


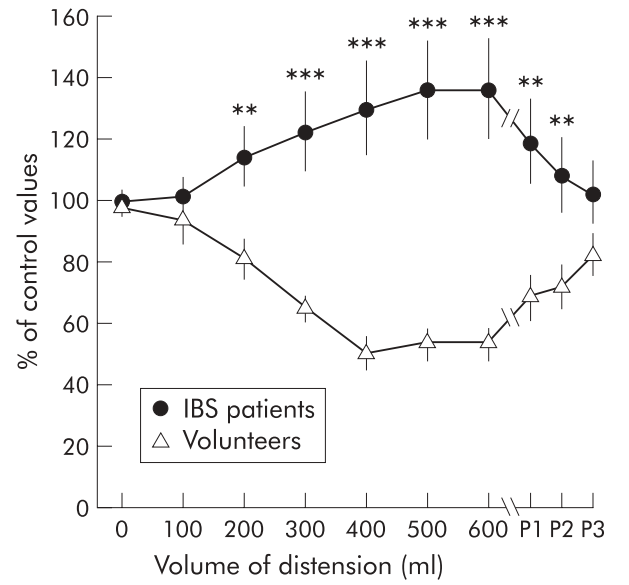

Figure 2 Cumulative results showing the effects of slow ramp distension on the somatic nociceptive cutaneomuscular flexion (RIII) reflex in healthy volunteers and irritable bowel syndrome (IBS) patients. The mean RIII reflex response recorded during each minute was expressed as a percentage of the mean value recorded during the three minute predistension control period (means (SEM)) and during the three minute post-distension period (P1, P2, P3). (A) In healthy volunteers, slow ramp distension induced a progressive decrease in the RIII with a progressive return to baseline after the end of distension. (B) In IBS patients, the effects of slow ramp distension were significantly different to those observed in volunteers as it induced progressive facilitation of the RIII reflex. Data are mean (SEM); ${ }^{* *} p<0.01 ;{ }^{* * *} p<0.001$.

$(600 \mathrm{ml})$. The RIII reflex responses were measured during the three minutes before distension (control period), during the continuous rectal distension period, and during the three minute post-distension period. RIII responses were averaged at one minute intervals and expressed as a percentage of the mean value obtained during the control period. Pressure was recorded at fixed intervals (every $50 \mathrm{ml}$ of distension). Sensations elicited by rectal distensions were scored as described above.

\section{Data analysis}

Results are expressed as mean (SEM). Statistical analysis of the effects of distensions on the RIII reflex were performed by two way analyses of variance, with Fisher's post hoc least significant difference test. During slow ramp distensions, when the pain threshold was reported before $600 \mathrm{ml}$, the corresponding RIII value recorded at the maximal tolerated volume was reported to subsequent distending volumes of up to $600 \mathrm{ml}$ in order to normalise data and to compare accurately both groups. Relationships between two variables were tested by the Kendall rank correlation. Wilcoxon's signed ranked test and the Mann-Whitney test were used for comparison of paired and unpaired data. A p value of $<0.05$ was considered significant.

\section{RESULTS}

Fourteen IBS patients and 10 healthy volunteers completed the study. Three IBS patients were excluded because they could not tolerate the electrical stimuli $(n=2)$ or because of a lack of stability of the reflex responses $(n=1)$.

In IBS patients, the mean daily intensity of abdominal pain during the week before the experiment was 29.9 (24.7) and the intensity of abdominal bloating was 48.5 (26.3). The intensity of abdominal pain and bloating on the experimental day were not significantly different from those reported during the pretest period.

The RIII reflex threshold was not different between IBS patients $\left(\begin{array}{ll}6.3 & (0.3) \mathrm{mA}\end{array}\right)$ and healthy volunteers (7.6 (0.4) $\mathrm{mA})(\mathrm{p}=0.3)$.

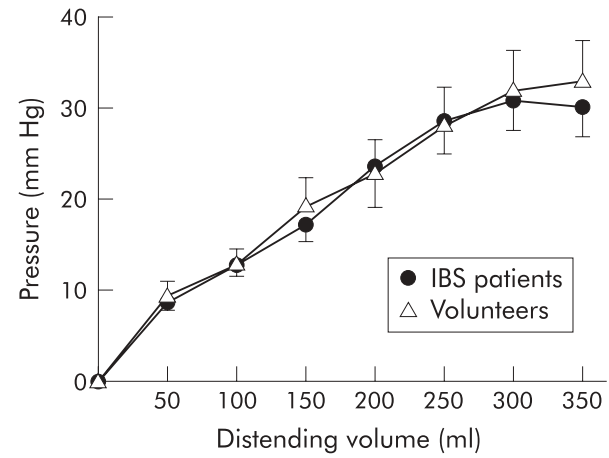

Figure 3 Curves showing the volume-pressure relationship during slow ramp distensions in healthy volunteers and irritable bowel syndrome (IBS) patients. No significant difference was observed between the groups. Data are mean (SEM).
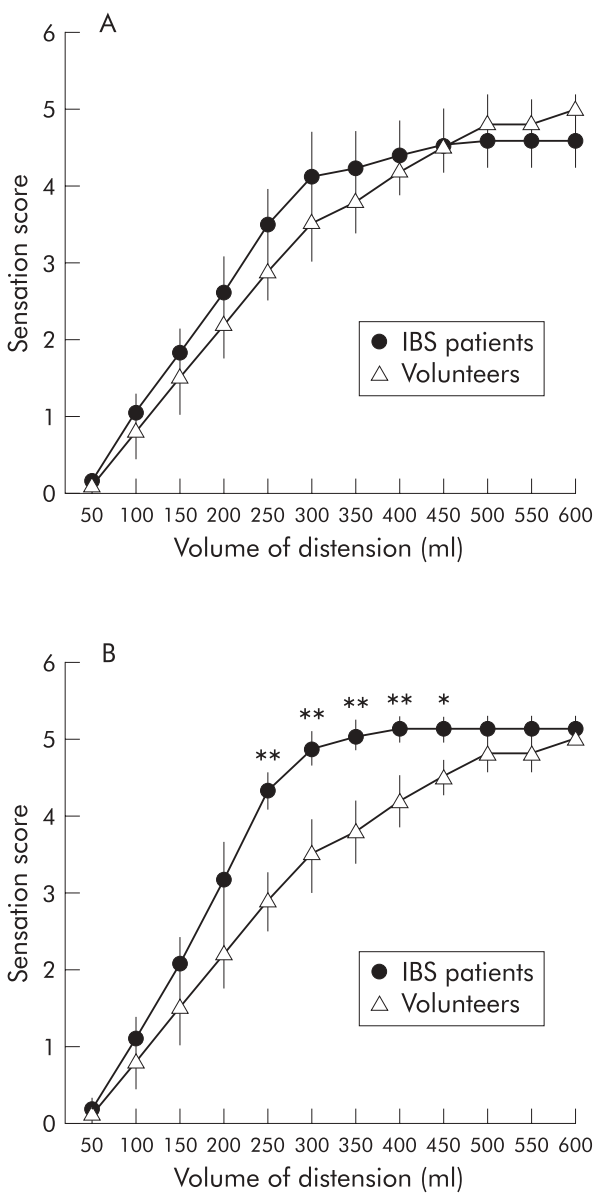

Figure 4 Curves showing the volume-sensation relationship during slow ramp distension. (A) Group comparisons did not show significant differences between healthy volunteers and irritable bowel syndrome (IBS) patients. (B) A significant increase in visceral sensation (that is, visceral hypersensitivity) was observed only in the subgroup of IBS patients $(n=10)$ showing facilitation of the somatic nociceptive cutaneomuscular flexion (RIII) reflex. ${ }^{*} p<0.05 ;{ }^{* *} p<0.01$.

\section{Effects of slow ramp distension}

Individual examples of the effects of slow ramp distensions on the RIII reflex in healthy volunteers and IBS patients are shown in fig 1 and cumulative data observed in the two groups are shown in fig 2 . In healthy volunteers, slow ramp distension induced a progressive inhibition of the reflex 

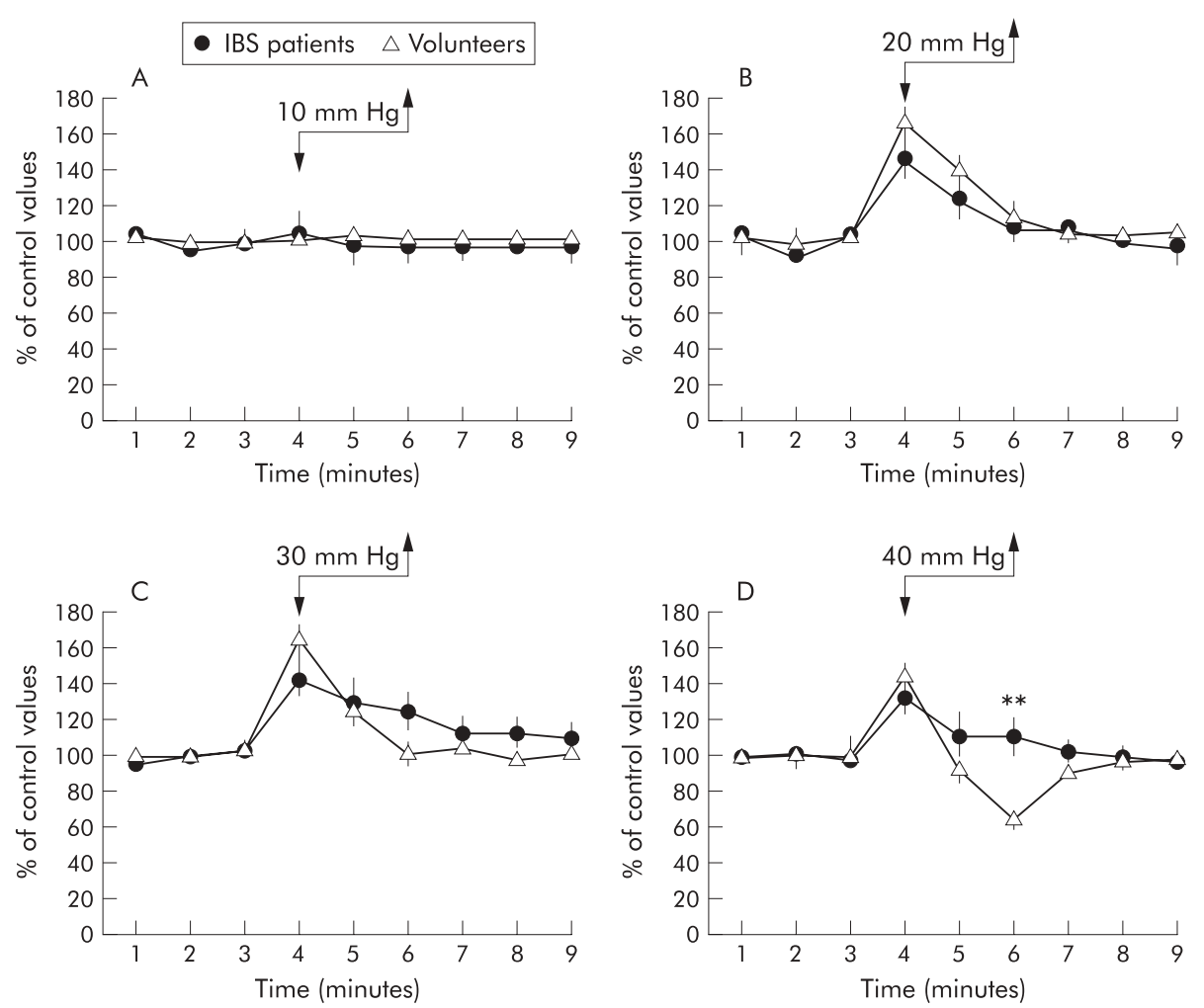

Figure 5 Cumulative data showing the effects of graded rapid rectal distensions ranging from 10 to $40 \mathrm{~mm} \mathrm{Hg}$ (A-D) on the somatic nociceptive cutaneomuscular flexion (RIII) reflex recorded from the lower limb in healthy volunteers and irritable bowel syndrome (IBS) patients. For each distension level, the mean RIII reflex response during each minute was expressed as a percentage of the mean value recorded during the three minute predistension control period. The three minute distension period is indicated by arrows. Data are mean (SEM); ${ }^{* *}<0.01$.

which peaked at the maximal volume of distension (61 (13)\% of control values). In contrast, progressive facilitation of the RIII reflex (139 (15)\% of pre-distension control values at the maximal volume of distension; $\mathrm{p}<0.01 v$ healthy volunteers) was observed during slow ramp distension in IBS patients. Individual analysis revealed that clear facilitatory effects (that is, increase in RIII responses by at least $20 \%$ ) were present in 10 of 14 IBS patients. Interestingly, seven of these 10 patients spontaneously reported an increase in the sensation evoked by electrical stimulation of the sural nerve during distension. In four IBS patients the effects of slow distension were similar to those observed in healthy volunteers.

Compliance curves were similar in the two groups (fig 3) as well as maximal volume of distension: 338 (92) $\mathrm{ml}$ in IBS patients versus 377 (118) $\mathrm{ml}$ in healthy volunteers.

The volume-sensation relationship was similar in the two groups (fig 4A). However, in the subgroup of 10 IBS patients demonstrating facilitation of the RIII reflex, sensations evoked by distension were significantly more pronounced in comparison with healthy volunteers (fig 4B). In this subgroup of patients, the RIII reflex threshold and compliance were not significantly different from healthy volunteers (results not shown). Finally, no relationship was found between the effects of slow ramp distension on the RIII reflex and clinical presentation of IBS patients (that is, pain intensity or abdominal bloating recorded the week before the experimental session as well as on the experimental day, duration of symptoms, and bowel frequency).

\section{Effects of rapid distensions}

The effects of rapid distensions on the reflex responses in healthy volunteers were different according to the level of

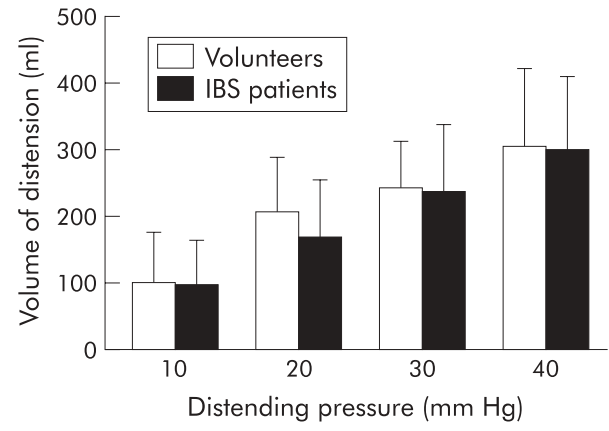

Figure 6 Volumes of distension during rapid rectal distensions (10, 20, 30 , and $40 \mathrm{~mm} \mathrm{Hg}$ ) in healthy volunteers and irritable bowel syndrome (IBS) patients. Differences were not significant.

distension. No significant change in the reflex was observed during the $10 \mathrm{mmHg}$ distension (fig 5A). An increase in the RIII responses was observed during the first min of distension of the 20 and $30 \mathrm{~mm} \mathrm{Hg}$ distensions (fig 5B, 5C). The $40 \mathrm{~mm} \mathrm{Hg}$ stepwise distension induced biphasic effects: facilitation of the RIII response during the first minute followed by inhibition during the third minute (fig 5D).

In IBS patients, the effects of the 10, 20, and $30 \mathrm{~mm} \mathrm{Hg}$ distension steps on the RIII responses were not significantly different from those observed in healthy volunteers. However, in contrast with healthy volunteers, no inhibition but facilitation of the RIII reflex was observed during the second and third minutes of the $40 \mathrm{~mm} \mathrm{Hg}$ distension $(\mathrm{p}<0.01)$ (fig 5D). 


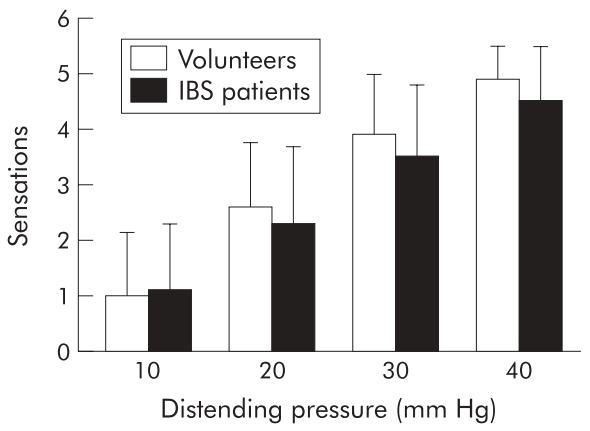

Figure 7 Perceived sensations during rapid rectal distension (10, 20, 30 , and $40 \mathrm{~mm} \mathrm{Hg}$ ) in healthy volunteers and irritable bowel syndrome (IBS) patients. Differences were not significant.

Volumes of distension during the 10, 20, 30, and $40 \mathrm{~mm} \mathrm{Hg}$ distension steps (fig 6), compliance curves (results not shown), and sensations evoked by rapid distensions (fig 7) were not significantly different between the two groups. The effects of rapid distensions on the RIII reflex were not related to the clinical presentation of IBS patients (that is, pain intensity or abdominal bloating recorded the week before the experimental session as well as on the experimental day, duration of symptoms, and bowel frequency).

\section{DISCUSSION}

In the present study, we found that rectal distensions induced facilitations of the RIII spinal nociceptive flexion reflex in a large subgroup of IBS patients. These results provide direct and objective arguments favouring the idea that visceral hypersentivity/hyperalgesia reported in IBS patients is associated with hyperexcitability of spinal nociceptive neurones.

The role of visceral hyperalgesia as a major pathophysiological determinant of functional bowel disorders was proposed some 30 years ago. This concept was initially based on the observation of a reduction in painful or discomfort thresholds during balloon distensions of the rectosigmoid in IBS patients and was expanded further to other functional gut disorders such as non-cardiac chest pain and functional dyspepsia. ${ }^{2}$ Other psychophysical results, such as enlarged referral patterns of pain during rectal distensions ${ }^{17}$ or reduction of the rectal pain threshold after repetitive stimulation of the sigmoid colon, ${ }^{18}$ also support this hypothesis. However, there is still very little objective evidence demonstrating alteration of visceral pain processing in IBS. A reduction in the latency of cortical responses evoked by electrical or mechanical rectal stimuli has been reported in some patients. ${ }^{4619}$ Interpretation of such results in terms of pain mechanisms is not unequivocal as these cortical responses are not specific for painful stimuli. In addition, it is not possible to determine whether changes in these potentials were due to peripheral and/or central sensory processing as their brain sources have not been clearly identified. Functional neuroimaging (that is, positron emission tomography or functional magnetic imaging) has also been used to investigate the cerebral regions involved in visceral hypersensitivity in functional gut disorders. However, the results of the studies conducted in IBS patients are too variable to allow any firm conclusions to be drawn. ${ }^{7}$

The RIII reflex is evoked specifically by painful stimuli and its threshold and amplitude are closely correlated with those of the concomitant pain sensation. ${ }^{8}$ It is considered a reliable and quantitative index of spinal transmission of nociceptive signals and recordings of this reflex have been used in numerous pathophysiological and pharmacological studies in patients and volunteers. ' Thus our results may represent the first objective evidence of alteration of spinal nociceptive processing in IBS patients. In these patients, facilitations of the RIII reflex were associated with an increase in the perception of rectal distension. They were also associated with an increase in cutaneous painful sensations due to electrical stimulation at the foot, which tends to rule out the possibility that the increase in the reflex responses was due to an action on the motor facet of the reflex (that is, facilitation of motoneurones in the ventral horn). It appears very likely that enhancement of the RIII reflex responses induced by rectal distensions was reflection of true facilitation of the spinal processing of viscerosomatic nociceptive information in a large subgroup of IBS patients. Facilitation of this somatic reflex, organised in the lumbosacral segments of the spinal cord, during visceral stimuli may be explained by hyperexcitability of spinal neurones receiving convergence of somatic and visceral sensory afferents which have long been demonstrated in electrophysiological studies in animals. ${ }^{20-22}$ In accordance with our results, increased responses to segmental somatic nociceptive stimuli (for example, thermal stimuli applied to the foot) have been reported in IBS patients. ${ }^{23}$ Such an association of visceral and segmental cutaneous hyperalgesia has also been observed in patients with non-cardiac chest pain. Sarkar and colleagues ${ }^{25}$ showed that acid infusion into the lower oesophagus resulted in a reduction in the electrical pain threshold in the upper part of the oesophagus as well as in the upper anterior chest wall.

At least two categories of mutually non-exclusive pathophysiological mechanisms can be proposed to explain such spinal hyperexcitability: (i) direct modification of the electrophysiological properties (that is, sensitisation) of spinal nociceptive neurones; and (ii) reduction of the inhibitory systems (that is, disinhibition) of spinal transmission of nociceptive information.

The "pathological" effects of visceral stimuli were mainly observed during slow ramp distensions while the effects of rapid distensions were similar to those observed in volunteers (except for the highest level of distension). Such differential effects depending on the mode of distension argue against sensitisation of spinal neurones which, on the contrary, would have resulted in facilitatory effects during both types of distension. The fact that the RIII reflex threshold was not different between healthy volunteers and IBS patients also tends to rule out the hypothesis of direct and permanent changes in the excitability of spinal neurones. Therefore, the hypothesis of failure of central inhibition in IBS patients appears more likely. Segmental and/or heterosegmental (that is, descending) systems that tonically or phasically modulate spinal transmission of somatic and visceral nociceptive signals have been described in both animals and humans. ${ }^{26}{ }^{27}$ Tonic modulatory systems of pain transmission were probably not altered in IBS patients as the RIII reflex thresholds were not different between patients and healthy volunteers. In contrast, facilitation of the reflex responses may be explained by a reduction in segmental and/or descending inhibitory controls, which are phasically triggered by visceral stimuli.

In conclusion, the present results suggest that our methodology may help to identify a subgroup of IBS patients with spinal hyperexcitability. This might be of particular interest as IBS is probably a heterogeneous and multifactorial condition, and clinical criteria, such as the Rome II criteria, do not allow discrimination between the relevant subgroups of patients. Further pathophysiological or pharmacological studies should aim at verifying whether the different subgroups of patients respond differently to new compounds acting on gut sensitivity. 


\section{ACKNOWLEDGEMENTS}

B Coffin and D Bouhassira were responsible for the design of the study, conduct of the experiments, analysis and interpretation of the data, and writing of the paper. J-M Sabaté and L Barbe contributed to the experiments. $\mathrm{R}$ Jian contributed to the design of the study and writing of the paper.

This study was supported by an unrestrictive grant funded by L'institut UPSA de la douleur and la Société d'Etude et de Traitement de la Douleur.

\section{Authors' affiliations}

B Coffin, D Bouhassira, J-M Sabaté, Service d'HépatoGastroentérologie, AP-HP Hopital Lovis Mourier, Colombes, France, and INSERM E-332, AP-HP, CHU Ambroise Paré, Boulogne-Billancourt and Université Versailles-Saint-Qentin, Versailles, France

L Barbe, Service d'Hépato-Gastroentérologie, AP-HP Hopital Louis Mourier, Colombes, France

R Jian, Service d'Hépato-Gastroentérologie, AP-HP Hopital Européen Georges Pompidou, Paris, France

\section{REFERENCES}

1 Thompson WG, Longstreth GF, Drossman DA, et al. Functional bowel disorders and functional abdominal pain. Gut 1999:45(suppl 2):1143-7.

2 Mertz H. Visceral hypersensitivity. Aliment Pharmacol Ther 2003;17:623-33.

3 Camilleri M. Testing the sensitivity hypothesis in practice: tools and methods, assumptions and piffalls. Gut 2002;51(suppl 1):i34-40.

4 Chan YK, Herkes GK, Badcock C, et al. Alterations in cerebral potentials evoked by rectal distension in irritable bowel syndrome. Am J Gastroenterol 2001;96:2413-17.

5 Malcolm A, Phillips SF, Kellow JE, et al. Direct clinical evidence for spinal hyperalgesia in a patient with irritable bowel syndrome. Am J Gastroenterol 2001;96:2427-31.

6 Sinhamahapatra P, Saha SP, Chowdhury A, et al. Visceral afferent hypersensitivity in irritable bowel syndrome-evaluation by cerebral evoked potential after rectal stimulation. Am J Gastroenterol 2001;96:2150-7.

7 Derbyshire SW. A systematic review of neuroimaging data during visceral stimulation. Am J Gastroenterol 2003;98:12-20.

8 Willer JC. Comparative study of perceived pain and nociceptive flexion reflex in man. Pain 1977;3:69-80.

9 Skljarevski V, Ramadan NM. The nociceptive flexion reflex in humans. Pain 2002;96:3-8
10 Bouhassira D, Danziger N, Attal N, et al. Comparison of the pain suppressive effects of clinical and experimental painful conditioning stimuli. Brain 2003; 126:1068-78

11 Bouhassira D, Chollet R, Coffin B, et al. Inhibition of a somatic nociceptive reflex by gastric distention in humans. Gastroenterology 1994;107:985-92.

12 Bouhassira D, Sabate JM, Coffin B, et al. Effects of rectal distensions on nociceptive flexion reflexes in humans. Am J Physiol 1998;275:G410-17.

13 Coffin B, Bouhassira D, Chollet R, et al. Effect of the kappa agonist fedotozine on perception of gastric distension in healthy humans. Aliment Pharmacol Ther 1996;10:919-25.

14 Coffin B, Farmachidi JP, Rueegg P, et al. Tegaserod, a 5-HT4 receptor partial agonist, decreases sensitivity to rectal distension in healthy subjects. Aliment Pharmacol Ther 2003;17:577-85.

15 Sabate JM, Coffin B, Jian R, et al. Rectal sensitivity assessed by a reflexologic technique: further evidence for two types of mechanoreceptors. Am J Physiol 2000;279:G692-9.

16 Drossman DA, Grant-Thompson W, Talley NJ, et al. Identification of subgroups of functionnal gastrointestinal disorders. Gastroenterol Int 1990;3: 159-72.

17 Mertz H, Naliboff B, Munakata J, et al. Altered rectal perception is a biological marker of patients with irritable bowel syndrome. Gastroenterology 1995; 109:40-52

18 Munakata J, Naliboff B, Harraf F, et al. Repetitive sigmoid stimulation induces rectal hyperalgesia in patients with irritable bowel syndrome. Gastroenterology 1997;1 12:55-63.

19 Rossel P, Pedersen P, Niddam D, et al. Cerebral response to electric stimulation of the colon and abdominal skin in healthy subjects and patients with irritable bowel syndrome. Scand J Gastroenterol 2001;36:1259-66.

20 Cervero F. Sensory innervation of the viscera: peripheral basis of visceral pain. Physiol Rev 1994;74:95-138.

21 Cervero F, Laird JM. Visceral pain. Lancet 1999;353:2145-8.

22 Ness TJ, Gebhart GF. Visceral pain: a review of experimental studies. Pain 1990;41:167-234

23 Chang L, Mayer EA, Johnson T, et al. Differences in somatic perception in female patients with irritable bowel syndrome with and without fibromyalgia. Pain 2000;84:297-307.

24 Verne GN, Robinson ME, Price DD. Hypersensitivity to visceral and cutaneous pain in the irritable bowel syndrome. Pain 2001;93:7-14

25 Sarkar S, Aziz Q, Woolf CJ, et al. Contribution of central sensitisation to the development of non-cardiac chest pain. Lancet 2000;356:1154-9.

26 Ren K, Dubner R. Descending modulation in persistent pain: an update. Pain 2002;100:1-6.

27 Fields HL, Basbaum AL. Central nervous system mechanisms of pain modulation. Central nervous system mechanisms of pain modulation. In, Wall, PD, Melzack R, eds. Textbook of pain Edinburgh, Churchill Livingstone, 1999:309-29. 